\title{
Growth-Mark Formation and Chronology of Two Neotropical Anuran Species
}

\author{
Federico Marangoni, ${ }^{1,2}$ Eduardo Schaefer, ${ }^{1}$ Rodrigo Cajade, ${ }^{1}$ and Miguel Tejedo ${ }^{3}$ \\ ${ }^{1}$ Department of Amphibian Ecology, Centro de Ecología Aplicada del Litoral (CECOAL-CONICET), C.C. 291, \\ 3400 Corrientes, Argentina \\ ${ }^{3}$ Department of Evolutionary Ecology, Estación Biológica de Doñana (C.S.I.C.), Avda. de María Luisa s/n, \\ Pabellón del Perú, 41013, Sevilla, Spain; E-mail: (MT) tejedo@ebd.csic.es
}

\begin{abstract}
Aвstract.-In amphibians from tropical or subtropical regions well-expressed growth marks in bone could be expected because of the existence of strong dry/wet seasons, but little evidence is available. Additionally, temporal patterns of growth-mark formation may be a function of genetic and environmental factors. We used skeletochronology and a laboratory experiment to tease apart both causal sources, establishing direct correspondence between an observed pattern of bone growth and the actual age of Ceratophrys cranwelli and Dermatonotus muelleri from the subtropical Argentinean Chaco. Also, we describe the growth by application of the von Bertalanffy model. Growth rates decreased in both species, tending to reach asymptotic values at age 8-10 months after metamorphosis. Both species clearly exhibited haematoxylinophilic lines of arrested growth (LAG) after one year growing in captivity. These results reinforce the hypothesis that in subtropical climates the LAG formation is ultimately caused by a general intrinsic (genetic) control.
\end{abstract}

Skeletochronology is based on the presence of cyclic and annular bone growth, which can be visualized in cross-sections of bone (Castanet, 1982; Castanet and Smirina, 1990). This method of counting the number of LAGs (lines of arrested growth) in cross-sections of phalanges obtained by toe clipping is the most commonly used tool for evaluating the age of amphibians and reptiles, providing an age estimate through nonlethal means (Castanet and Smirina, 1990; Tejedo et al., 1997; Eden et al., 2007). Annual periodicity in deposited growth marks has been demonstrated in a large number of amphibians from temperate zones, where winter quiescence slows growth during this part of the year (reviews: Castanet et al., 1993; Smirina, 1994; Castanet et al., 2003). These studies support the hypothesis of Castanet et al. (1993) that LAG formation is ultimately caused by a genetically based, circannual rhythm, which under natural conditions becomes synchronized with, and reinforced by, the seasonal cycle. Therefore, both intrinsic and extrinsic factors may cause LAG formation and periodicity. Very few skeletochronological studies have been carried out on amphibians from tropical or subtropical regions where growth is constrained less by temperature but where growth may be subjected to periodicity through wet/dry seasons (Kumbar and Pancharatna, 2002). Thus, we could expect that the presence of well-expressed growth marks in such climates would suggest that both intrinsic cycles (Morrison et al., 2004) and extrinsic factors have an influence on the rhythm of osteogenesis throughout the year. Furthermore, it is still not clear whether these growth marks are formed annually and whether they can be regarded as year rings to determine the age of these tropical amphibian species. In addition, supplementary nonannual LAGs have been observed occasionally (e.g., double LAGs or

${ }^{2}$ Corresponding Author. Present Address: FCEQy N, Universidad Nacional de Misiones, Félix de Azara 1552, 3300 Posadas, Misiones, Argentina. E-mail: fmarangoni@ebd.csic.es 
annuli lines, Tejedo et al., 1997; Sinsch et al., 2007), which necessitates comparing the estimated age based on skeletochronology with the known age of the individual, before ages can be assigned confidently. One approach to differentiate the intrinsic/environmental sources of variation determining the formation and periodicity of LAGs is by raising individuals of known age in the laboratory where potential environmental factors can be controlled.

Ceratophrys cranwelli and Dermatonotus muelleri are distributed in the warm temperate climate of the Chaco region of Argentina to Bolivia, Brazil, and Paraguay. The Chaco region of Argentina has a subtropical climate, with a long dry season with rainfall mainly during the summer months (mean annual precipitation $51,200-500 \mathrm{~mm}$, east to west, respectively; Barrio, 1980). Both species are explosive breeders: mating coincides with seasonal rainfall, from October to January (Peltzer and Lajmanovich, 2007). Outside of the breeding season, adults remain buried underground. Cocoon formation is characteristic in C. cranwelli (McClanahan et al., 1976) that aids conservation of body water during dry conditions. Dermatonotus muelleri burrows into the ground near waterlogged soils or under termite mounds near the breeding sites (Cei, 1980).

The main goal of this study was to determine whether well-expressed growth marks are formed in these anurans when raised in the laboratory where growth is not constrained by highly variable environmental conditions. This would help to determine whether formation is genetically or environmentally based. A secondary goal was to use individuals of known age to determine whether skeletochronology is a valid method for estimating age in these two subtropical anurans. Finally, we describe the growth over the study period by application of the von Bertalanffy model to determine the time required to achieve the asymptotic minimum size for reproduction.

Materials and Methods

On 5 December 2006, 20 tadpoles of C. cranwelli (stage 30; Gosner, 1960) and 30 tadpoles of D. muelleri 
TABLE 1. Body mass (BM), snout-vent length (SVL), right hind length (HLR), and head width (HW). Values are mean 61 SD. * Significantly higher $(P>0.001$, Kolmogorov-Smirnov Test) values at final compared with initial values. Sm 5 average maximal body size estimated from von Bertalanffy equations. Values are mean $6 \mathrm{SE}$.

\begin{tabular}{|c|c|c|c|c|c|c|}
\hline & \multicolumn{3}{|c|}{ Ceratophrys cranwelli } & \multicolumn{3}{|c|}{ Dermatonotus muelleri } \\
\hline & Initial (N 5 7) & Final (N 5 6) & $\mathrm{Sm}$ & Initial (N 5 9) & Final (N 5 8) & $\mathrm{Sm}$ \\
\hline $\mathrm{BM}(\mathrm{g})$ & 1.6960 .25 & $80.28610 .82 *$ & 120.3369 .92 & 0.5760 .22 & $14.4662 .82 *$ & 17.2262 .52 \\
\hline SVL (mm) & 26.0161 .69 & 88.96 3.46* & 94.9361 .10 & 19.1163 .03 & $52.8463 .08 *$ & 55.7860 .51 \\
\hline HLR (mm) & 29.4261 .9 & $91.5463 .52 *$ & 97.4661 .15 & & & \\
\hline $\mathrm{HW}(\mathrm{mm})$ & 14.960 .77 & $42.6262 .29 *$ & 44.8660 .56 & & & \\
\hline
\end{tabular}

(stage 39) were captured in a temporary pond near Laguna Yema (Formosa province, Argentina, 24u20944.50S, 61u06954.50W). Tadpoles were brought to the laboratory and kept together in 45-liter plastic trays until metamorphosis. The first metamorphs (defined as the emergence of the first forelimb; stage 42) of C. cranwelli and D. muelleri were found on 12 and 15 December 2006, respectively. Each metamorph (C. cranwelli: N 5 7; D. muelleri: N 5 9) was kept separately in 1-liter plastic trays in the laboratory and maintained under natural temperature (mean 6 1SD $\min 521.965 .12 \mathrm{uC}$, $\max 525.0964 .52 \mathrm{uC}$ ) and photoperiod (day length varied from 1045-1330 h, because the laboratory has two glass windows on one side that allowed natural light to enter the room throughout the day). Relative humidity ranged from $35-63 \%$. Ceratophrys cranwelli and D. muelleri were fed on cichlids and termites (Gymnogeophagus sp., 2.436 $0.92 \mathrm{~g}$, Cortaritermes fulviceps $1.5660 .24 \mathrm{~g}$ ) twice a week. The snout-vent length (SVL) and right hindlimb length (HLR) were measured by placing each toad on laminated graph paper (accuracy $61 \mathrm{~mm}$ ). Head width (HW) was measured to the nearest $0.01 \mathrm{~mm}$, using a digital caliper, and body mass (BM) to the nearest $0.01 \mathrm{~g}$, using an Acculab 221 electronic balance (Acculab. Scales, Titusville, NJ). All measurements were recorded once per week. After one year, in the second week of December 2007, the terminal phalange of the third digit of the right forefoot was clipped and preserved in $70 \%$ alcohol for age determination by skeletochronology. Additionally, on 13 October 2007, three adult males of D. muelleri were captured in a temporary pond in Corrientes province (27u55928.40S, 57u29934.50W). This allowed comparison between their adult body size and age with the toads reared in the laboratory conditions. The methods described in detail by Castanet (1982) and Castanet and Smirina (1990) for age determination by skeletochronology were followed. The third toe of each animal was selected, washed in water for one hour, decalcified in 5\% nitric acid for 30-45 min, and rinsed in tap water overnight. Transverse sections (16 mm thick) were cut using a cryostat microtome, stained with Ehrlich's haematoxylin for $10 \mathrm{~min}$ and rinsed in tap water for one hour. Finally, we selected 5-10 transverse sections, the ones with the smallest marrow cavity and the thickest cortical bone, and mounted them on microscope slides with a 50\% glycerin and 50\% alcohol solution. Representative sections for each individual were photographed using a digital camera (Olympus, FE-120, Olympus Imaging America, Inc.) and viewed and measured using the computer package Image-Pro Plus Version 1.1 (Media Cybernetics, 1993-94). The analysis and interpretation of the LAGs was done under an ordinary light microscope followed by photomicrography. Age was determined using two observers (FM and RC) who counted LAG independently to prevent any bias, considering the outer perimeter of the bone as a LAG (Patón et al., 1991). The longest and shortest perpendicular axes of each LAG and perimeter were measured in each of two diaphyseal sections per specimen examined, following the methods of Hemelaar (1985). Axis measurements were multiplied together and the square root of the product calculated (average diameter of each LAG). Growth was estimated by application of the von Bertalanffy growth model (von Bertalanffy, 1938). Application of this model in skeletochronology studies has already been described in detail by Hemelaar (1988) and Miaud et al. (2001), and has been successfully used on anurans and urodeles. The von Bertalanffy growth model was fitted to the average growth curve using the leastsquare procedure. All statistical analyses were performed using the STATISTICA 6.0 statistical package (StatSoft, Tulsa, OK, 2001).

\section{RESULTS}

Six of seven metamorphs of C. cranwelli and eight of nine of $D$. muelleri kept in the laboratory conditions survived until the termination of the experiment (one year later). Morphological traits increased significantly in both species compared to the corresponding values at the beginning of the experiment (Table 1). All toads clearly exhibited two haematoxylinophilic lines of arrested growth after one year growing in captivity (Fig. 1). We also observed other more weakly expressed lines between the marrow cavity and the first LAG (Fig. 1A). These false lines are typical of young individuals whose growth is not continuous but vary in their intensity, as has been observed in Bufo and Triturus (Patón et al., 1991; Tejedo et al., 1997; Sinsch et al., 2007; U. Sinsch, pers. comm.). The size of the marrow cavity, metamorphosis line, and the LAGs were recorded for each individual and species (Table 2). The line of metamorphosis was visible in only two C. cranwelli and four D. muelleri specimens. Nevertheless, in those specimens in which it had been replaced during the formation of endosteal bone (C. cranwelli $566 \%$, D. muelleri 5 50\%), the first LAG remained unaffected (Table 2). The $\mathrm{R}^{2}$-values (C. cranwelli: $R^{2}$ : 0.977 , D. muelleri: $R^{2}: 0.947$ ) and asymptotic standard errors of the estimated parameters (Table 1, Fig. 2) indicate that the model fits quite 



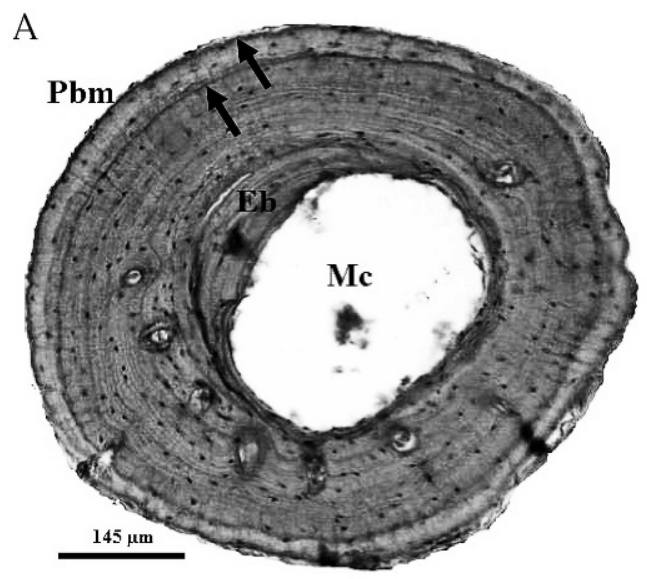

$\mathrm{B}$

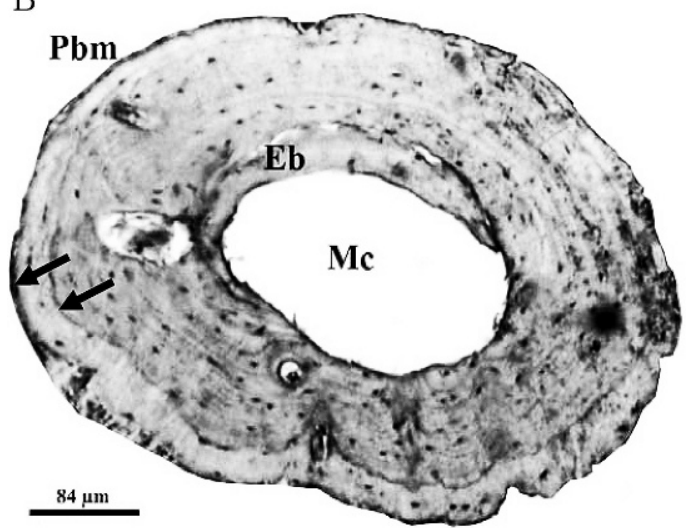

C

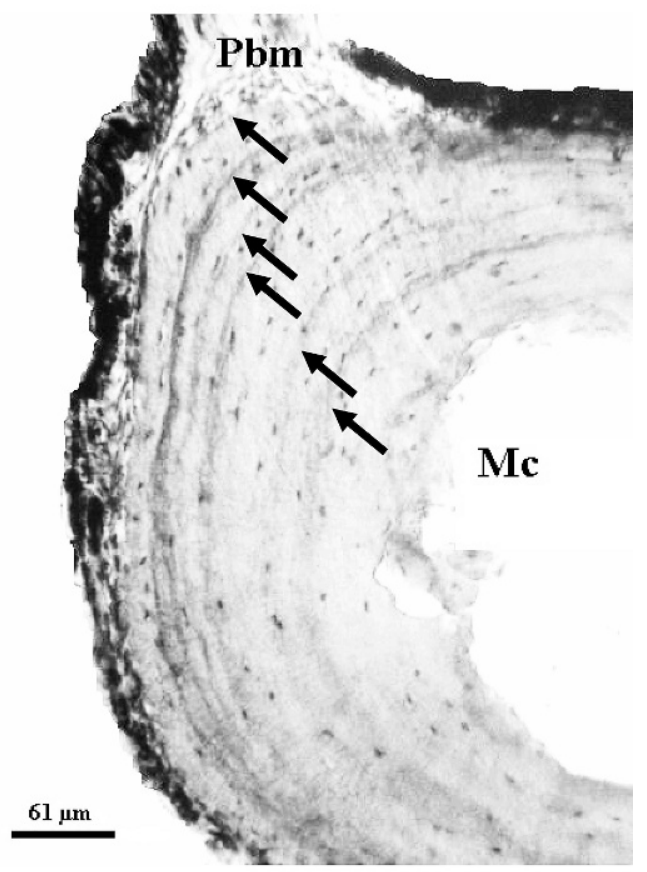

Fig. 1. Cross-sections of the third toe, stained by Ehrlichs hematoxylin of two toad species reared in laboratory conditions for one year: Ceratophrys cranwelli (A) and Dermatonotus muelleri (B). Arrows indicate LAGs. Adult of D. muelleri captured in the wild (C), showing six LAGs (Male, BM 522.4 g, SVL 560 mm). Mc 5 marrow cavity, Eb 5 endosteal bone, Pbm 5 periosteal bone margin.

well. The predicted maximal body size (Sm) was always larger than averaged values measured (Table 2). However, some individuals at the end of the experiment reached the predicted asymptotic size in BM (C. cranwelli: individual \#6 BM max $_{1}: 119.44$ g, D. muelleri: individual \#2 $\mathrm{BM}_{\max } 515.32 \mathrm{~g}$ ), and even their SVL went beyond the Sm-value (C. cranwelli: individual \#6 SVL $\max 597 \mathrm{~mm}$, D. muelleri: individual \#2 SVL $\max 59 \mathrm{~mm}$ ). These results suggest that laboratory-raised toads of both species reached the asymptotic value. In addition, the predicted Sm was lower with respect to mean and maximum adult body size of D. muelleri captured in the wild (mean 6 SD 5 BM: 21.363 .56 g, N 5 3; SVL: 62.6661 .69 mm, N 5 3; $\mathrm{BM}_{\max } 526.36 \mathrm{~g} ; \mathrm{SVL}_{\max } 565 \mathrm{~mm}$ ). We found well-defined growth marks in only one of the three adults captured. We clearly recorded six LAGs in the bone of this toad (Fig. 1C).

\section{Discussion}

This is one of the few studies to have been carried out on skeletochronology in amphibians from tropical or subtropical regions and reports the first evidence of the correspondence between an observed pattern of bone growth and actual age of $\mathrm{C}$. cranwelli and $\mathrm{D}$. muelleri. Our results show that both species clearly exhibit haematoxylinophilic LAGs after they were maintained in the laboratory under their natural photoperiod, temperature, and regular food for one year. The presence of well-expressed growth marks in these toads, in which growth was not constrained by environmental conditions, supports the hypothesis that, for these species inhabiting a highly seasonal subtropical climate, the LAG formation is ultimately caused by a general intrinsic (genetic) control (e.g., Kumbar and Pancharatna, 2002; Morrison et al., 2004).

Two main difficulties have to be overcome to consider skeletochronology as a reliable technique to assign age confidently. First, and ideally, age estimates based on skeletochronology should be compared with individuals of a known age. The second complexity in assigning age underlies the process of identification and interpretation of the LAGs. The processes of bone resorption and reconstruction can destroy LAGs, which may lead to underestimating the age of the toads (but see Hemelaar, 1985; Eggert and 
TABLE 2. Bone growth of Dermatonotus muelleri and Ceratophrys cranwelli. Mc 5 marrow cavity, Ml 5 metamorphosis line, LAG 5 line of arrested growth. * $5 \mathrm{Ml}$ replaced during the formation of endosteal bone.

\begin{tabular}{|c|c|c|c|c|c|c|c|c|}
\hline \multirow[b]{3}{*}{ Individual } & \multicolumn{8}{|c|}{ Bone growth (mm) } \\
\hline & \multicolumn{4}{|c|}{ Dermatonotus muelleri } & \multicolumn{4}{|c|}{ Ceratophrys cranwelli } \\
\hline & $\mathrm{Mc}$ & $\mathrm{Ml}$ & LAG1 & LAG2 & Mc & $\mathrm{Ml}$ & LAG1 & LAG2 \\
\hline 1 & 222.7 & 346.38 & 541.5 & 613 & 177.5 & 335 & 635 & 762 \\
\hline 2 & 246.2 & $*$ & 482.2 & 541.3 & 265 & $*$ & 512.5 & 572.5 \\
\hline 3 & 185.7 & $*$ & 516 & 593.9 & 105 & 217 & 537.5 & 625 \\
\hline 4 & 278 & $*$ & 500 & 569 & 285 & $*$ & 522.5 & 585 \\
\hline 5 & 268.9 & 320.1 & 495 & 565 & 285 & $*$ & 520 & 580 \\
\hline 6 & 450 & $*$ & 629 & 722.4 & 457.5 & $*$ & 607.5 & 732.5 \\
\hline 7 & 220.9 & 342 & 437 & 490.4 & & & & \\
\hline 8 & 243.03 & 360 & 425 & 501.6 & & & & \\
\hline
\end{tabular}

Guyétant, 2003). However, supplementary growth marks (e.g., double lines, Castanet et al., 1993; Tejedo et al., 1997), which are histologically indistinguishable from LAGs with an annual periodicity, can lead to overestimation of age (Sinsch et al., 2007). In this study, complete endosteal removal of the inner LAGs was never observed (Table 2); therefore, resorption did not influence age estimates. In addition, false lines weakly expressed in some individuals, between the marrow cavity and the first LAG, were clearly visible, and easily identified (Fig. 2). Nevertheless, the fact that some individuals, in spite of the small sample, exhibited variation in the bone growth pattern by expressing two LAGs after one year growing in captivity, casts doubt on the reliability of skeletochronology in estimating age in these two Neotropical species. Complementary information about actual activity and quiescent periods of the animal in natural

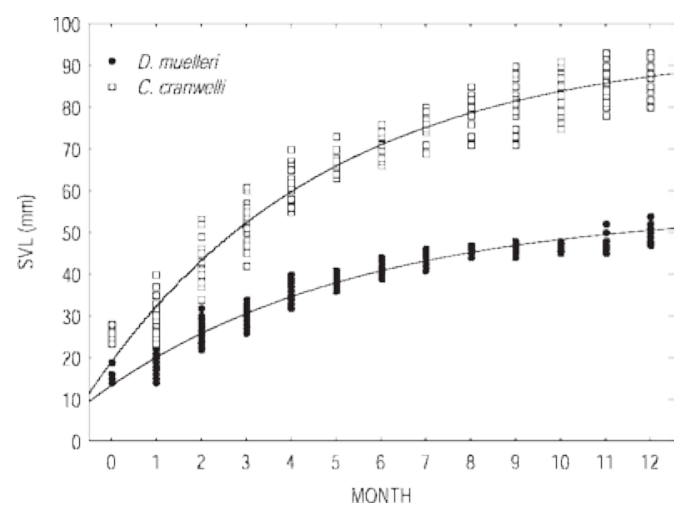

Fig. 2. Growth curves and estimated parameters from von Bertalanffy equations, for body growth (SVL) of Ceratophrys cranwelli and Dermatonotus muelleri. Model: SVL 5 Sm 2 [(Sm 2 So) 3 exp (2 K 3 month)]. Sm 5 average maximal body size, $\mathrm{S}_{0}$ 5 body size at metamorphosis, $\mathrm{k} 5$ growth coefficient, defining the shape of the growth curve. A high value of $\mathrm{k}$ indicates that $\mathrm{Sm}$ will be achieved soon. Dermatonotus muelleri: SVL 5 (55.7889) 2 \{[(55.7889) 2 (13.26)] 3 exp [2 (0.173556) 3 month]\}, C. cranwelli: SVL 5 (94.9328) $2\{[(94.9328) 2$ (18.9265)] 3 exp [2 (.192302) 3 month]\}. populations with information based on a markrecapture method (Halliday and Verrell, 1988) will be necessary to check and reinforce our results.

Postmetamorphic growth in amphibians exhibits an indeterminate pattern with an exponential period that decreases considerably after the onset of reproduction with the attainment of an asymptotic size (Hemelaar, 1988). This change in the shape of the growth rate curve may be explained as a consequence of reallocating energy once sexual maturity is achieved, from somatic growth to energy storage and reproduction (Stearns, 1992). The two species studied here show the growth rate pattern described above, with an exponential period at first, which eventually becomes asymptotic at one year of growth (Fig. 2). This asymptotic size also corresponds to the time at which the first LAG in the bone was recorded. Furthermore, we noted calling activity after 301 and 305 days of metamorphosis (on 10 and 16 October 2007, C. cranwelli and D. muelleri, respectively). Moreover, the mean phalangeal diameter of D. muelleri reared in the laboratory (mean $6 \mathrm{SD}$, PER: $574.5674 .08 \mathrm{~mm}, \mathrm{~N} 5$ 8) was similar to that found in the five-year-old adult captured in the field $(650.5 \mathrm{~mm})$. Our measurements (Table 1) and the available adult body size information for C. cranwelli (min and max 5 SVL: 110-125, Peltzer and Lajmanovich [2007]; mean 6 SD 589.38611 .10 mm, N 5 18, Barrio [1980]; mean 5 $79 \mathrm{~mm}$ Scott and Aquino [2005]) and D. muelleri (min and max 5 SVL: 40-50 mm Cei [1980]), suggest that the toads of our study have achieved the minimum size for reproduction after only one year of growth in captivity.

Acknowledgments.-We thank C. Gonzalez for invaluable help with the fieldwork. U. Sinsch and M. Iturra-Cid reviewed earlier versions of the manuscript and provided useful comments that helped improve it. We thank also to $\mathrm{B}$. Machado for correcting the English draft of this manuscript. We acknowledge L. Rossi and A. Ibañ ez for their assistance in the sample processing. We thank $M$. Sanchez-Negrette and $M$. Montenegro for providing the corresponding permits and facilities to work at the Cátedra de Patología General y Sistemática and Cátedra de Histología y Embriología, Facultad de Ciencias Veterinarias, Universidad Nacional del Nordeste. The authors have complied with all applicable Institutional Animal Care guidelines, and all required state and federal permits have been obtained. 


\section{Literature Cited}

Barrio, A. 1980. Una nueva especie de Ceratophrys (Anura, Ceratophryidae) del Dominio Chaqueño. Physis Sección C 39:21-30.

Bertalanffy, L. von. 1938. A quantitative theory of organic growth. Human Biology 10:181-213.

CAstanet, J. 1982. Recherches sur la croissance du tissu osseux des reptiles. Application: la mé thode squelettochronologique. Unpubl. PhD diss., Université Paris 7, Paris.

Castanet, J., and E. Smirina. 1990. Introduction to the skeletochronological method in amphibian and reptiles. Annales des Sciences Naturelles, Zoologie et Biologie Animale 11:191-196.

Castanet, J., H. Francillon-Vieillot, F. J. Meunier, and A. DE Ricqlès. 1993. Bone and individual aging. In B. K. Hall (ed.), Bone Growth. Vol. 7, pp. 245-283. CRC Press, Boca Raton, FL.

Castanet, J., H. Francillon-Vieillot, A. De Ricqlè S, and L. Zylberberg. 2003. The skeletal histology of the Amphibia. In H. Heatwole and M. Davies (eds.), Amphibian Biology. Vol. 5, pp. 1598-1683. Surrey Beatty and Sons, Chipping Norton, New South Wales, Australia.

Cei, J. M. 1980. Amphibians of Argentina. Monitore Zoologico Italiana (N.S.). Monografia 2:1-609. EdEN, C. J., H. H. Whiteman, L. Duobinis-Gray, and S. A. Wissinger. 2007. Accuracy Assessment of Skeletochronology in the Arizona Tiger Salamander (Ambystoma tigrinum nebulosum). Copeia 2007: 471-477.

Eggert, C., AND R. GuYÉtant. 2003. Reproductive behaviour of Spadefoot Toads (Pelobates fuscus): daily sex ratios and males' tactics, ages, and physical condition. Canadian Journal of Zoology 81:46-51.

Gosner, K. L. 1960. A simplified table for staging anuran embryos and larvae with notes on identification. Herpetologica 16:183-190.

Halliday, T. R., and P. Verrell. 1988. Body size and age in amphibians and reptiles. Journal of Herpetology 22:253-265.

Hemelaar, A. 1985. An improved method to estimate the number of year rings resorbed in phalanges of Bufo bufo (L.) and its application to population from different latitudes and altitudes. AmphibiaReptilia 6:323-341.

. 1988. Age, growth and other population characteristics of Bufo bufo from different latitudes and altitudes. Journal of Herpetology 22:369-388.
Kumbar, S. M., and K. Pancharatna. 2002. Annual growth layers in the phalanges of the Indian Skipper Frog Rana cyanophlyctis (Schn.). Copeia 2002:870-872.

McClanahan, L., H. Shoemakevr, and R. Rubal. 1976. Structure and function of the cocoon of a ceratophryd frog. Copeia 1976:179-185.

Miaud, C., F. Andreone, A. Ribéron, S. De Michelis, V. Clima, J. Castanet, H. Francillon-Vieillot, and R. GuYÉTANT. 2001. Variations in age, size at maturity and gestation duration among two neighbouring populations of the Alpine Salamander (Salamandra lanzai). Journal of Zoology 254:251-260,

Morrison, C., J-M. Hero, and J. Browning. 2004. Altitudinal variation in the age at maturity longevity, and reproductive lifespan of anurans in subtropical Queensland. Herpetologica 60: 34-44.

Patón, D., A. Jurranz, E. Sequeros, R. Perez-Campo, M. Lopez-Torres, and G. B. De Quiroga. 1991. Seasonal age and sex structure of Rana perezi assessed by skeletochronology. Journal of Herpetology 25: 389-394.

Peltzer, P. M., and R. C. Lajmanovich. 2007. Amphibians. In M. H. Iriondo, J. C. Paggi, and M. J. Parma (eds.), The Middle Paraná River: Limnology of a Subtropical Wetland, pp. 327-340. SpringerVerlag, Berlin, Germany.

ScotT, N. J., JR., AND A. L. Aquino. 2005. It's a frog eat frog world in the Paraguayan Chaco: food habits, anatomy, and behavior of the frog-eating anurans. In M. A. Donnelly, B. I. Crother, C. Guyer, M. H. Wake, and M. E. White (eds.), Ecology and Evolution in the Tropics: A Herpetological Perspective, pp. 243-259. University of Chicago Press, Chicago.

Sinsch, U., N. Oromi, and D. Sanuy. 2007. Growth marks in Natterjack Toad (Bufo calamita) bones: histological correlates of hibernation and aestivation periods. Herpetological Journal 17:129-137.

Smirina, E. M. 1994. Age determination and longevity in amphibians. Gerontology 40:133-146.

Stearns, S. C. 1992. The Evolution of Life Histories. Oxford University Press, Oxford.

Tejedo, M., R. Reques, and M. Esteban. 1997. Actual and osteochronological estimated age of Natterjack Toads (Bufo calamita). Herpetological Journal 7:81-82.

Accepted: 25 November 2008. 